\title{
Aleochara pseudochrysorrhoa, a new species from southern Brazil (Coleoptera: Staphylinidae: Aleocharinae), with a complete checklist of Neotropical species of the genus
}

\author{
Edilson Caron ${ }^{1}$; Kleber M. Mise ${ }^{1} \&$ Jan Klimaszewski ${ }^{2}$ \\ ${ }^{1}$ Laboratório de Sistemática e Bioecologia de Coleoptera, Departamento de Zoologia, Universidade Federal do Paraná. \\ Caixa Postal 19020, 81531-980, Curitiba, Paraná, Brasil. E-mail: caron@ufpr.br; klebermise@yahoo.com.br \\ 2 Natural Resources Canada, Canadian Forest Service, Laurentian Forestry Centre. 1055 du PEPS, P.O. Box 10380 Ste-Foy, \\ Québec, Québec, Canada G1V 4C7. E-mail:jklimaszewski@cfl.forestry.ca
}

\begin{abstract}
The ectoparasitoid habit of larvae and the active predatory habit of adults of Aleochara Gravenhorst, 1802 established this group as natural fly regulators and important for ecological studies, biological control programs and forensic entomology. In the current study, a new aleocharine species, Aleochara pseudochrysorrhoa sp. nov., from southern Brazil, is described and its natural history is briefly discussed. The species has a robust body, uniformly dark-brown to black with apex of abdomen rust-brown, median lobe of male with expanded bulbus, sclerites of internal sac forming complex arrangement, and female with spermatheca L-shaped. Aleochara pseudochrysorrhoa sp. nov. may be considered to be closely related to the species belonging to the Iustrica group. A complete checklist of Neotropical species of Aleochara is also provided. Nomenclatural problems are also discussed. Aleochara lateralis Erichson, 1839 is a junior primary homonym of A. Iateralis Heer, 1839, and is replaced by the available name Aleochara bonariensis Lynch, 1884. A new name, Aleochara newtoni nom. nov., is proposed to replace Maseochara (= Aleochara) duplicata Sharp, 1883, which is a junior secondary homonym of A. duplicata Erichson, 1839.
\end{abstract}

KEY WORDS. Forensic entomology; new name; rove beetle; South America; taxonomy.

RESUMO. Aleochara pseudochrysorrhoa, nova espécie do sul do Brasil (Coleoptera: Staphylinidae: Aleocharinae), com uma lista completa das espécies Neotropicais do gênero. Os hábitos ectoparasitóide da larva e predador ativo dos adultos de Aleochara Gravenhorst, 1802 faz deste um grupo regulador natural de moscas e importante para estudos ecológicos, programas de controle biológico e entomologia forense. No presente estudo, uma nova espécie de Aleocharinae, Aleochara pseudochrysorrhoa sp. nov., do sul do Brasil é descrita e notas de sua histórianatural são brevemente discutidas. A espécie possui corpo robusto, uniformemente castanho-escuro a preto e ápice do abdome castanho-avermelhado, o macho possui lobo médio com base bulbosa e uma complexa estrutura dos escleritos no saco-interno, e a fêmea possui espermateca em forma de “L”. Aleochara pseudochrysorrhoa sp. nov. pode ser considerada fortemente relacionada com as espécies do grupo lustrica. Uma lista completa das espécies Neotropicais de Aleochara é também apresentada.Também são discutidos problemas nomenclaturais. Aleochara lateralis Erichson, 1839 é homônimo primário júnior de A. lateralis Heer, 1839, e é substituído por Aleochara bonariensis Lynch, 1884. Um novo nome, Aleochara newtoni nom. nov., é proposto para substituir Maseochara (= Aleochara) duplicata Sharp, 1883, que é homônimo secundário júnior de A. duplicata Erichson, 1839.

PALAVRAS-CHAVE. América do Sul; entomologia forense; estafilinídeos; nome novo; taxonomia.

Adults and larvae of Aleochara Gravenhorst, 1802 are found in fly-infested habitats such as animal droppings, carrion, and decaying organic matter such as forest litter, mushrooms, fermented fruit and decaying seaweed (KLIMAszewski \& JANSEN 1993). The adults prey on eggs, larvae and pupae of cyclorraphous Diptera, and larvae feed on pupae inside puparia (Peschke \& Fuldner 1977, Klimaszewski 1984, Klimaszewski \& Jansen 1993, Maus et al. 1998). The females lay their eggs in sites infested with fly larvae, and the newly emerged first-instar larvae hunt for dipteran pupae, and feed on them until pupation takes place, either in the soil or within host puparia (Kuimaszewski 1984, Maus et al. 1998).

The ectoparasitoid habit of larvae and the active predatory habit of adults of Aleochara established this group as natural fly regulators and important for ecological studies (JoNASSON 1994), biological control programs (Jones 1967, BROMAND 1980, WRight 
et al. 1989, JoNASSON 1990) and forensic indicators (SouzA \& Linhares 1997, TABOR et al. 2005, Mise et al. 2007). The extensive natural history of Aleochara is published in KLIMASZEwSKI \& JANSEN (1993) and a complete list of host records in Maus et al. (1998).

Aleochara comprises some 437 species worldwide (NEwTON $\&$ Thayer 2005) of which about 81 species occur in the Neotropical region. Since the revision of the Nearctic species of Aleochara (KuImaszewski 1984), several attempts have been made to study type specimens of Aleochara species from the Neotropical region (Klimaszewski \& GÉnier 1986, KuImaszewski et al. 1987, KuIMaszewsKi 1989, Klimaszewski \& Maus 1999, Maus 1998, 2001). Furthermore, several species have been described (PACE 1987, 1990, 2000, Klimaszewski 1990, Klimaszewski \& Ashe 1992, Maus 1999, 2000). The goal of the present study is to describe a new species of Aleochara (s. str.) from southern Brazil, with brief comments on its probable phylogenetic relationships and notes on its natural history. In addition, a complete checklist of Neotropical species of Aleochara, with synonymic list, type locality, subgeneric affiliation and currently known distribution are also provided. Nomenclatural problems (two homonyms) are also discussed.

\section{MATERIAL AND METHODS}

Dried specimens were macerated in boiling water for five minutes and then segments VIII-X were separated from the abdomen, dissected and cleared in boiling $10 \% \mathrm{KOH}$ for two minutes. The dissections were carried out under a Zeiss Stemi SV6 stereoscopic microscope and the drawings were made using a Zeiss Standard 20 optical microscope with attached drawing tube. Photographs were taken using a Leica MZ 16 stereoscopic microscope with a Leica DFC 500 digital camera. The images were captured with IM 50 (Image Manager) computer software and combined using Auto-Montage Pro 5.03.0040 image processing software. The final drawings and photographs were produced using Adobe Photoshop software, version 7.0.

Measurements were taken using the micrometric ocular in a Wild M5 stereoscopic microscope. The following abbreviations were used: (BL) body length (from anterior margin of head to posterior margin of tergite VIII); (EW) elytra width (maximum); (PL) pronotum length (maximum); (PW) pronotum width (maximum); (EL) elytra length (maximum).

The terminology used in the text is adopted from BlaCKWELder (1936) and KLIMASZEWSKI (1984).

Specimens are deposited in the Coleção de Entomologia Pe J.S. Moure, Departamento de Zoologia, Universidade Federal do Paraná, Curitiba, Brazil (DZUP); Instituto Nacional de Pesquisas da Amazônia, Manaus, Brazil (INPA); Museu Nacional do Rio de Janeiro, Rio de Janeiro, Brazil (MNRJ); Museu Paraense Emílio Goeldi, Belém, Brazil (MPEG); Museu de Zoologia, Universidade de São Paulo, São Paulo, Brazil (MZSP); Museu de Ciências Naturais da Fundação Zoobotânica do Rio Grande do Sul, Porto Alegre, Brazil (MCNZ); American Museum of Natural History, New York, USA (AMNH); The Natural History Museum, London, England (BMNH); Centro de Estudios en Zoología,
Entomología, Universidad de Guadalajara, Guadalajara, Mexico (CZUG); Field Museum of Natural History, Chicago, USA (FMNH); the Insectarium R. Martineau, Laurentian Forestry Centre, Quebec, Canada (LFC); Museo de La Plata, La Plata, Argentina (MLP); and Museo de Historia Natural, Universidad Nacional Mayor de San Marcos, Lima, Peru (MUSM).

Label data of type material are in brackets ([), the data from each label are enclosed within double quotes (" ") and a forward slash (/) separates lines.

In the checklist, subgenera and species are arranged alphabetically, with the original names and the synonyms listed chronologically under valid specific names.

The Neotropical region as treated here includes Mexico, Central and South America. Extra-Neotropical distributions are listed by regions based on NewTon et al. (2005).

\section{RESULTS}

\section{Aleochara (Aleochara) pseudochrysorrhoa Caron, Mise \& Klimaszewski sp. nov.}

\section{Figs 1-10}

Type material. Holotype (male) [“Curitiba - PR - Brazil/ Centro Politécnico/2526'45"S 49¹3'58”W/919meters/15-XII2005/Mise, K.M. col.", "Pig carcass/Modified Shannon/Trap" (DZUP). Paratypes, 453 with the same labels as the holotype, except the date: 01-XI-2005 (9 DZUP), 02-XI-2005 (4 DZUP), 03-XI-2005 (8 DZUP), 05-XI-2005 (16 MNRJ), 06-XI-2005 (26 DZUP), 07-XI-2005 (2 DZUP), 10-XI-2005 (6 DZUP), 11-XI-2005 (3 DZUP), 12-XI-2005 (14 DZUP), 13-XI-2005 (15 MLP), 14-XI2005 (15 DZUP), 15-XI-2005 (18 AMNH), 16-XI-2005 (6 DZUP), 17-XI-2005 (5 DZUP), 19-XI-2005 (11 DZUP), 20-XI-2005 (6 DZUP), 21-XI-2005 (7 DZUP), 22-XI-2005 (8 DZUP), 23-XI-2005 (15 DZUP), 24-XI-2005 (21 MZSP), 26-XI-2005 (3 DZUP), $27-$ XI-2005 (2 DZUP), 28-XI-2005 (3 DZUP), 29-XI-2005 (22 LFC), 30-XI-2005 (12 DZUP), 01-XII-2005 (15 MPEG), 02-XII-2005 (5 DZUP), 04-XII-2005 (4 DZUP), 05-XII-2005 (18 BMNH), 06XII-2005 (5 DZUP), 07-XII-2005 (1 DZUP), 09-XII-2005 (5 DZUP), 10-XII-2005 (11 DZUP), 11-XII-2005 (3 DZUP), 13-XII2005 (6 MUSM), 14-XII-2005 (9 INPA), 15-XII-2005 (11 DZUP), 16-XII-2005 (31 FMNH), 17-XII-2005 (8 DZUP), 18-XII-2005 (1 DZUP), 19-XII-2005 (3 DZUP), 20-XII-2005 (16 MCNZ), 22-XII2005 (1 DZUP), 23-XII-2005 (4 DZUP), 24-XII-2005 (5 DZUP), 25-XII-2005 (2 DZUP), 26-XII-2005 (1 DZUP), 28-XII-2005 (3 DZUP), 29-XII-2005 (2 DZUP), 30-XII-2005 (11 DZUP), 31-XII2005 (15 CZUG).

Diagnosis. Body robust, uniformly dark-brown to black with apex of abdomen rust-brown (Figs 1 and 2). Dorsal surface glossy, pubescent, and setigerous pores impressed. Median lobe of male with expanded bulbus (Fig. 8), short tubus and acute apex, without subapical-ventral projection in lateral view, and sclerites of internal sac forming complex arrangement (Figs 8 and 9: a-f). Female with spermatheca L-shaped, capsule spherical and stem elongate (Fig. 10). 

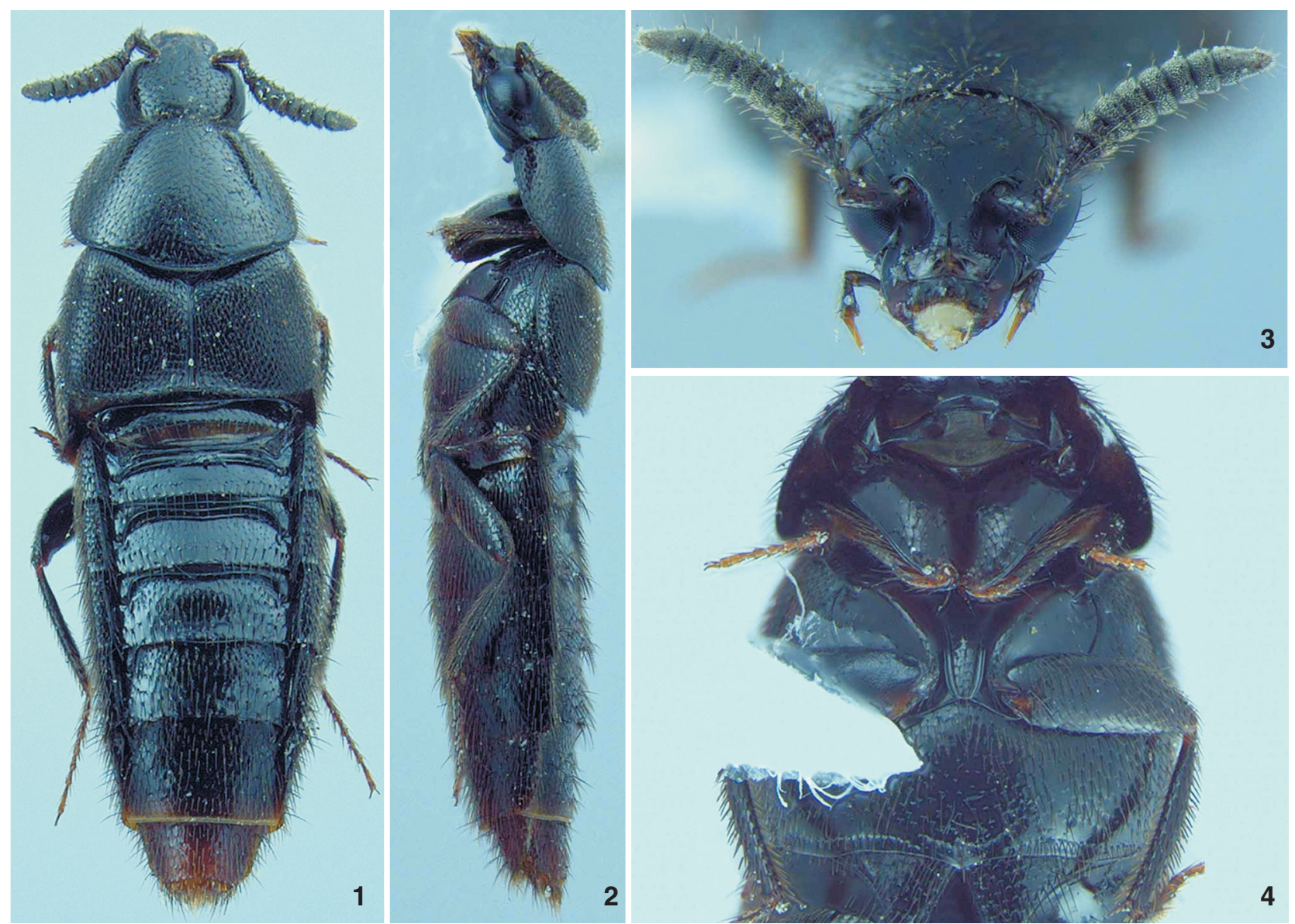

Figures 1-4. Aleochara pseudochrysorrhoa sp. nov.: (1) dorsal view; (2) lateral view; (3) head, frontal view; (4) meso and metaventrite, ventral view.

Description. BL: 5.42-7.75 mm; EW: 1.58-2.25 mm.

Body compact and robust (Fig. 1), elytra slightly broader than pronotum; uniformly dark-brown to black, with antennae, maxillary palpi, labial palpi, legs and apex of abdomen (apical half of abdominal segment VII and the segments VIII$\mathrm{X}$ ) rust-brown (Figs 1-3). Dorsal surface glossy; pubescent, appearing yellowish-brown in artificial light; setigerous pores impressed. Head approximately circular, as wide as long; frons triangularly produced and sharply pointed; pubescence directed anterad and mediad; eyes expanded, twice the length of temples; antennae with antennomere 4 slightly transverse and antennomeres 5-10 each twice as wide as long (Fig. 3); maxilla with palpus 4 -articulated and with minute pseudopalpomere on apex of last palpomere, which is approximately the same length as palpomere 3. Pronotum transverse (PW/PL: 1.44), with posterior margin broadly arcuate; densely pubescent, with pubescence straight and directed posterad in the midline of the disc and posterad and laterad elsewhere. Mesoventrite broadly rounded posteriorly, reaching posterior portion of mesocoxae
(Fig. 4). Elytra as long as pronotum (EL/PL: 1.02); elytra about twice as wide as long (EW/EL: 1.74); densely pubescent, with straight pubescence directed posterad. Hind wings well developed. Abdomen gradually narrowed posteriorly, glossy, and with sparse pubescence directed posterad; first three visible terga deeply transversely impressed in the anterior region, and fourth tergum shallowly impressed anteriorly (Fig. 1). Male. Tergite VIII sparsely pubescent, bearing a few prominent setae in posterior half; posterior margin serrate and slightly emarginate (Fig. 5). Sternite VIII with sparse pubescence, a few prominent macrosetae in posterior half; posterior margin truncate. Tergite IX divided and with asymmetrical ventral struts. Tergite X, with posterior margin slightly emarginated. Aedeagus: median lobe with expanded bulbus (Fig. 8); short tubus and acute apex, without subapical-ventral projection in lateral view; sclerites of internal sac forming a complex arrangement (Figs 8 and 9: a-f); parameres well developed, exceeding the apex of median lobe (Fig. 7). Female. Tergite and sternite VIII similar to those of male, except for lack of serration on posterior margin of

Revista Brasileira de Zoologia 25 (4): 827-842, December, 2008 

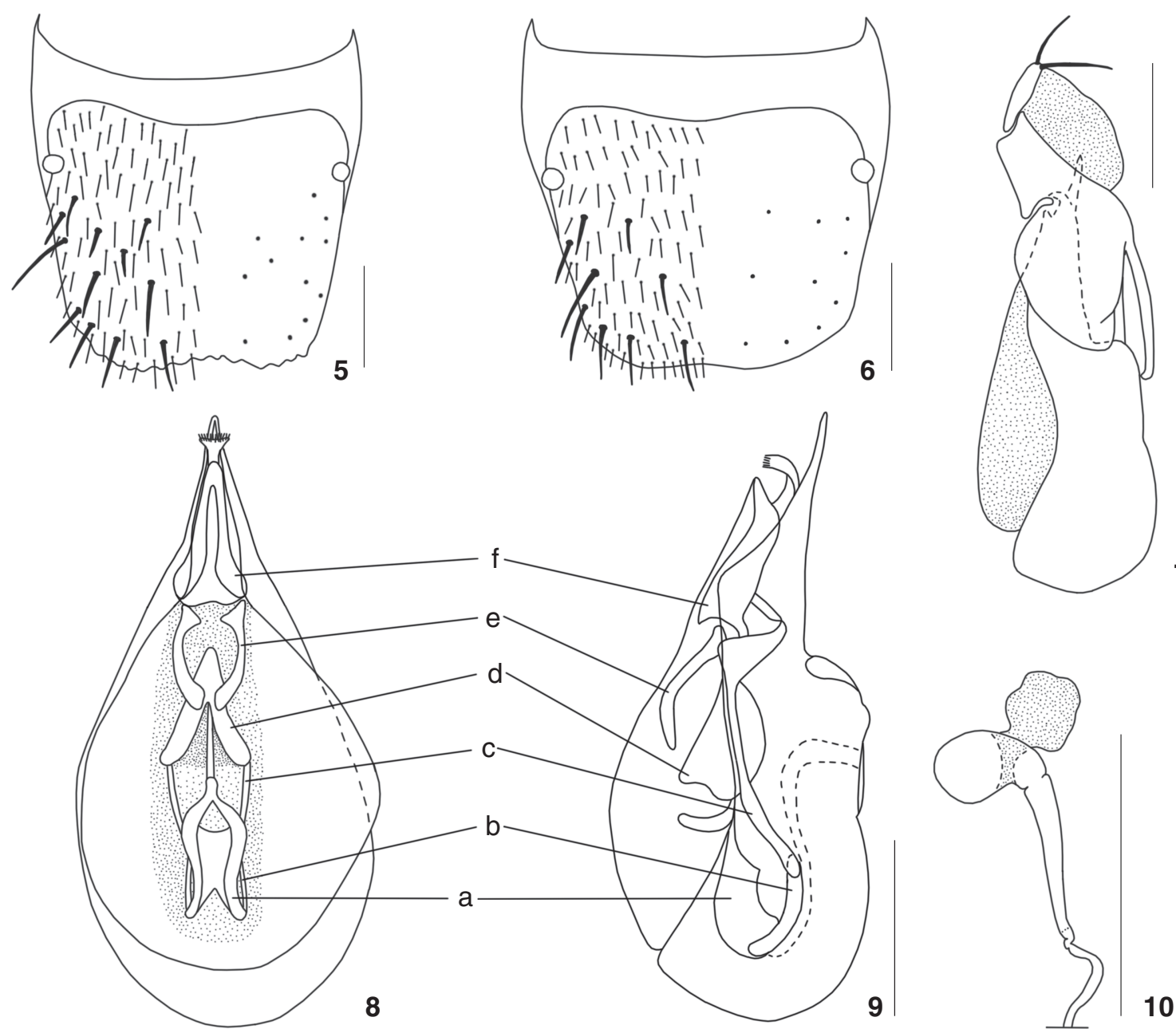

Figures 5-10. Aleochara pseudochrysorrhoa sp. nov.: (5) tergite VIII (right setae removed), male, dorsal view; (6) tergite VIII (right setae removed), female, dorsal view; (7) aedeagus (median lobe and paramere), lateral view; (8) median lobe, dorsal view; (9) median lobe, lateral view; (10) spermatheca. Scale: $0.25 \mathrm{~mm}$.

tergite VIII (Fig. 6), and posterior margin broadly rounded with short and dense setae; tergite IX without ventral struts. Spermatheca somewhat L-shaped, with narrowly spherical capsule and elongate stem (Fig. 10).

Etymology. The species name derives from the prefix pseudo- (false) added to the epithet chrysorrhoa (as in Aleochara chrysorrhoa Erichson, 1839).

Distribution. Southeastern Brazil (Paraná, Curitiba).

Remarks. The subgenus Aleochara was redefined by KLIMASZEWSKI (1984) and the most distinctive features of this taxon are the strongly transverse antennomeres 5-10 (Fig. 3), strongly elongate last maxillary palpomere, stout and broad body without dorsal microsculpture, evenly pubescent pronotum (Fig. 1), and usually wide and non-carinated mesoventrite (Fig. 4). Our species belongs to the lustrica species group, defined by KLIMASZEWSKI (1984) and diagnosed by the antennomere 4 transverse (Fig. 3), antennomeres 5-10 strongly transverse and at least twice as wide as long, glossy integument (Fig. 1), moderately to densely pubescent pronotum, large eyes (at least twice as long as temples) (Fig. 2), median lobe of the aedeagus with expanded bulbus and reduced triangularly shaped tubus (Figs 8 and 9) and spermatheca with sac-shaped capsule and elongate narrow stem (Fig. 10).

Due to these similarities $A$. pseudochrysorrhoa sp. nov. may be considered to be closely related to the following species: $A$. breviuscula Erichson, 1839; A. boliviana Pace, 1990; A. centralis Sharp, 1883; A. chrysorrhoa; A. curtula (Goeze, 1777); A. hidalgo Klimaszewski, 1990; A. bonariensis Lynch, 1884 (= A. lateralis Erichson, 1839); A. lustrica Say, 1832; and A. pseudolustrica Klimaszewski et al., 1990.

Aleochara pseudochrysorrhoa sp. nov. however, differs from the species listed above by the median lobe with acute apex and without subapical-ventral projection (Fig. 9), different ar- 
rangement of sclerites of internal sac (Figs 8 and 9); and spermatheca with spherical capsule (Fig. 10).

In the general form, A. pseudochrysorrhoa sp. nov. is very similar to A. chrysorrhoa, from which it can be distinguished mainly by the shape of the spermatheca: A. pseudochrysorrhoa sp. nov. has a spermatheca with a narrowly spherical capsule in contrast with elongate capsule of $A$. chrysorrhoa (KLIMASZEwSKI et al. 1987: 251, Fig. 1). The male of $A$. chrysorrhoa is unknown (KLIMASZEWSKi et al. 1987).

Natural history notes. Aleochara pseudochrysorrhoa sp. nov. was collected on carcasses of domestic pig, Sus scrofa (Linné, 1758) (Artyodactila, Suidae), in Curitiba, Brazil. This species was referred to as Aleochara sp.1 in Mise et al. (2007), and it was the most abundant species collected by hand sampling and in modified Shannon traps and one of the first species of Coleoptera to arrive on carcasses.

Aleochara pseudochrysorrhoa sp. nov. was collected yearround, from "fresh" to "butyric fermentation" decompositional stages as defined by Bornemissza (1957). In spring and summer this species has greater importance to the post mortem interval (IPM) determination, since it occurs throughout the entire period, in contrast to autumn and winter, in which it occurs periodically, colonizes, departs and recolonizes the carcass, making the intervals of the first or second colonization difficult to determine (Mise et al. 2007).

\section{Checklist of Neotropical species of Aleochara}

\section{Aleocharinae, Fleming, 1821 Aleocharini Fleming, 1821 Aleochara Gravenhorst, 1802}

Type species: Staphylinus curtulus Goeze, 1777: 730 (= Staphylinus fuscipes sensu Gravenhorst, 1802) (SMEtana 2004). For review of literature of the genus, see KLIMAszewski (1984), Smetana (2004) and Gouix \& Klimaszewski (2007). For redescription of the genus, see KlimaszewsKi (1984).

\section{Subgenus Aleochara Gravenhorst, 1802}

For redescription of the subgenus, see KLIMASzEwsKi (1984).

Aleochara atra (Solier, 1849: 348) (as Mecorhopalus ater). [Type locality: "Santiago y en Valdivia" (Solier 1849)]. Note: Fairmaire \& Germain (1861) cited this species as Aleochara atra (Solier, 1849). The specific name is an adjective and was changed to the feminine spelling to agree with the gender of the generic name (Alfred F. Newton, from FMNH, pers. comm.).

Aleochara humilis Fauvel, 1866: 286. [Type locality: "Santiago" (FAuvel 1866)]. Remarks: synonymized by PACE (1990) (error: Fauvel, 1865).

Aleochara gravenhorsti Blackwelder, 1944: 167. [Type locality: "Santiago y en Valdivia" (Solier 1849)]. Note: proposed as a new name for Aleochara atra (Solier, 1849) (not Gravenhorst, 1806).
Aleochara paleoatra Pace, 2000: 404. [Type locality: "Santiago y en Valdivia" (Solier 1849)]. Note: Proposed as a new name for Aleochara atra (Solier, 1849) (not Gravenhorst, 1806). Remarks. We propose to keep A. atra (Solier, 1849) as the valid name, because ERICHSON (1837) already transferred $A$. atra Gravenhost, 1806 to the Tachyusa Erichson, 1837 and it is presently in the Thinonoma Thomson, 1859. Since these homonyms have not been congeneric for a long time, and the two replacement names have not come into use, $A$. atra (Solier) can be retained as valid by provision of Article 59.3 of the International Code of Zoological Nomenclature (ICZN 1999), and the replacement names proposed by BLACKWELder (1944) and PACE (2000) are unnecessary (Alfred F. Newton, from FMNH, pers. comm.). Subgeneric assignment. Aleochara (PACE 2000).

Distribution. Chile, Argentina (Scheerpeltz 1972, Pace 2000).

Aleochara auricoma Sharp, 1876: 70. [Type locality: "Ega" (SHARP 1876)].

Subgeneric assignment. Aleochara (BERNHAUER \& SCHEERPELTZ 1926).

Distribution. Brazil (Blackwelder 1944).

Aleochara boliviana Pace, 1990: 172. [Type locality: "Bolivia, Yungas de La Paz" (PACE 1990)].

Subgeneric assignment. Aleochara (PACE 1990).

Distribution. Ecuador, Bolivia, Peru (PACE 1990).

Aleochara bonariensis Lynch, 1884: 70. [Type locality: "Chacabuco" (Lynch 1884)].

Aleochara lateralis Erichson, 1839: 161 (not Heer, 1839). [Type locality: "Aragua" (Kuimaszewski et al. 1987)]. Note: $A$. lateralis Erichson, 1839 is a junior primary homonym of A. lateralis Heer, 1839, and must be rejected (Alfred F. Newton, from FMNH, pers. comm.). The relative priority of the Erichson and Heer publications was discussed by NewTON \& THAYER (1992).

Remarks. Aleochara bonariensis Lynch, 1884, was cited as a junior synonym of $A$. lateralis Erichson, 1839, by BERNHAUER $\&$ Scheerpeltz (1926). We follow Article 23.3.5 of the International Code of Zoological Nomenclature (ICZN), which requires that "... if a name in use for a taxon is found to be unavailable or invalid it must be replaced by the next oldest available name from among its synonyms" (ICZN 1999).

Subgeneric assignment. Aleochara (KLIMAszewski et al. 1987).

Distribution. Mexico, Colombia, Venezuela, Suriname, French Guiana, Brazil, Paraguay, Argentina, Cuba (Solsky 1872, NewTon et al. 2005).

Aleochara breviuscula Erichson, 1839: 161. [Type locality:

"Colombia" (KuIMASZEwsKi et al. 1987)].

Subgeneric assignment. Aleochara (KLimaszewski et al. 1987).

Distribution. Colombia (Newton et al. 2005). 
Aleochara bruchi Bernhauer, 1927: 263. [Type locality: "Republic Argentina, Caspinchango Catamarca" (KLIMASzewsKI \& Maus 1999)].

Subgeneric assignment. Aleochara (KLIMAszewski \& Maus 1999).

Distribution. Argentina (KLIMASZewski \& MAus 1999).

Aleochara cameroni Bernhauer \& Scheerpeltz, 1926: 776. [Type locality: "Grenada, St. Vincent" (CAMERon 1923)]. Note: Replacement name for A. bugnioni Cameron, 1923, preoccupied by A. curtula bugnioni Fauvel, 1901.

Aleochara bugnioni Cameron, 1923: 389 (not Fauvel, 1901). [Type locality: "Grenada, St. Vincent" (CAMERon 1923)].

Remarks. The above list of synonyms is from BernHAUER \& SCHEERPELTZ (1926).

Subgeneric assignment. Aleochara (Bernhauer \& SCHEerpeltZ 1926).

Distribution. Grenada, Saint Vincent Island (Saint Vincent and the Grenadines) (BLACKWELDER 1944).

Aleochara cayennensis Laporte, 1835: 136. [Type locality: “Cayenne" (LAPORTE 1835)].

Subgeneric assignment. Aleochara (Bernhauer \& SCHEERPELTZ 1926).

Distribution. French Guiana (BLACKWELder 1944).

Aleochara centralis Sharp, 1883: 146. [Type locality: "Costa Rica, Irazu 6000 to $7000 \mathrm{ft}$ " (KLIMASZEWSKi 1984)].

Aleochara stygialis Sharp, 1887: 775. [Type locality: "Mexico,

Zapotlan, Colima" (Kumaszewski 1984)].

Remarks. The above list of synonyms is from KLIMASZEwSKI (1984).

Subgeneric assignment. Aleochara (KLIMASzEwsKi 1984).

Distribution. Mexico, Guatemala, Costa Rica, Panama; Nearctic (Arizona, USA) (Navarrete-Heredia et al. 2002).

Aleochara chrysorrhoa Erichson, 1839: 160. [Type locality: "Brazil" (Kumaszewski et al. 1987)]. 1987).

Subgeneric assignment. Aleochara (KLIMASzEwski et al.

Distribution. Brazil (KumaszewsKi et al. 1987).

Aleochara cribricollis Fairmaire \& Germain, 1861: 413. [Type locality: "Santiago" (Fairmaire \& Germain 1861)].

Subgeneric assignment. Aleochara (Bernhauer \& SCHEERPeltZ 1926).

Distribution. Peru, Chile (Solsky 1872; Blackwelder 1944).

Aleochara curtula (Goeze, 1777: 730) (as Staphylinus curtulus). [Type locality: "France" (KLIMASzewsKi 1984)].

Staphylinus fuscipes Gravenhorst, 1802: 92 [Type locality: not given in Gravenhorst (1802)].

Staphylinus brachypterus Fourcroy, 1785: 167. [Type locality: "probably in France" (Klimaszewski 1984)].

Staphylinus limbatus Fabricius, 1801: 600. [Type locality: "Germany" (KlimaszewsKi 1984)].
Aleochara brevis Heer, 1839: 315. [Type locality: "Switzerland, Aigle" (Kumaszewski 1984)].

Aleochara puncticeps Thomson, 1860: 248. [Type locality: "Sweden, Gotheborg" (Kuimaszewski 1984)].

Aleochara discoidea Sharp, 1874: 7. [Type locality: "Japan" (KLIMASZEWSKi 1984)].

Aleochara discordia Duvivier, 1883: 99. [Type locality: "Japan" (Klimaszewski 1984)]. Note: misspelling for $A$. discoidea Sharp, 1874.

Aleochara curtula bugnioni Fauvel, 1901: 90. [Type locality: "Colombia, Sierra Nevada" (KLimaszewski 1984)].

Remarks. The above list of synonyms is from GouIx \& KLIMASZEWSKI (2007).

Subgeneric assignment. Aleochara (KLIMASzEwsKi 1984).

Distribution. Colombia, French Guiana, Peru, Bolivia, Brazil, Chile, Grenada, Saint Vincent Island (Saint Vincent and the Grenadines); Nearctic, Palearctic, Oriental, Afrotropical regions (Fauvel 1901, Newton et al. 2005).

Aleochara funeralis Motschulsky, 1858: 236. [Type locality: "Colombie" (Motschulsky 1858)].

Subgeneric assignment. Aleochara (Bernhauer \& SCHEERPeltz 1926).

Distribution. Colombia (Newton et al. 2005).

Aleochara hidalgo Klimaszewski, 1990: 174. [Type locality: "Mexico, Hidalgo, Tlanchinol, Alt. 1550m" (KuIMaszewski 1990)].

Subgeneric assignment. Aleochara (Klimaszewski 1990).

Distribution. Mexico, Costa Rica (Navarrete-Heredia et al. 2002).

Aleochara hilaris Sharp, 1887: 776. [Type locality: "Panama, Bugaba" (SHARP 1887)].

Subgeneric assignment. Aleochara (Bernhauer \& SCHEERPELTZ 1926).

Distribution. Panama (BLACKWELDER 1944).

Aleochara lata Gravenhorst, 1802: 186. [Type locality: "North America (America septentrionalis)" (KLIMASzewsKi 1984)].

Subgeneric assignment. Aleochara (KLIMAszewsKi 1984).

Distribution. Mexico, Peru, Chile; Nearctic and Paleartic regions (Solsky 1872, Navarrete-Heredia et al. 2002).

Aleochara lustrica Say, 1832: 55. [Type locality: "USA, Pennsylvania" (KuIMaszewsKi 1984)].

Aleochara pauper Sharp, 1883: 147. [Type locality: "Mexico, Jalapa" (KLimaszewski 1984)].

Aleochara serrata Sharp, 1883: 147. [Type locality: "Mexico, Tehuantepec" (KLIMaszewski 1984)].

Aleochara texana Casey, 1906: 137. [Type locality: "USA, Texas" (Klimaszewski 1984)].

Aleochara fusicornis Casey, 1906: 138. [Type locality: "USA" (KLimaszewski 1984)].

Aleochara sternalis Casey, 1906: 138. [Type locality: "USA, New York" (KLIMASzewski 1984)]. 
Aleochara algonquina Casey, 1906: 139. [Type locality: "USA, Ohio, Cincinnati" (KLimaszewski 1984)].

Aleochara medialis Casey, 1906: 140. [Type locality: “USA, New York" (KLIMASZEWSKI 1984)].

Remarks. The above list of synonyms is from KLIMASzEWSKI (1984).

Subgeneric assignment. Aleochara (KLIMASZEwski 1984).

Distribution. Mexico, Brazil, Chile; Nearctic region (PACE 1999, Navarrete-Heredia et al. 2002).

Aleochara mundana Sharp, 1876: 71. [Type locality: "Pará, Tapajos and Ega" (SHARP 1876)].

Subgeneric assignment. Aleochara (BERNHAUER \& SCHEERPELTZ 1926).

Distribution. Brazil (BLACKWELDER 1944).

Aleochara nigrocaerulea Cameron, 1923: 389. [Type locality: "Jamaica" (CAmeron 1923)].

Subgeneric assignment. Aleochara (BERNHAUER \& SCHEERPELTZ 1926).

Distribution. Jamaica (BLACKWELDER 1944).

Aleochara obscuricollis Say, 1832: 55. [Type locality: "Mexico" (SAY 1832)].

Subgeneric assignment. Aleochara (BERNHAUER \& SCHEERPELTZ 1926).

Distribution. Mexico (Navarrete-Heredia et al. 2002).

Aleochara parvicollis Bernhauer, 1904: 241. [Type locality: "Marcapata, Peru, 1000-2000m" (Klimaszewski \& Maus 1999)].

Subgeneric assignment. Aleochara (KuIMaszewski \& Maus 1999).

Distribution. Colombia, Peru (Newton et al. 2005).

Aleochara prisca Sharp, 1876: 69. [Type locality: "Ega" (SHARP 1876)].

Subgeneric assignment. Aleochara (Bernhauer \& SCHEERPELTZ 1926).

Distribution. Brazil (BLACKWELDER 1944).

Aleochara pseudochrysorrhoa Caron, Mise \& Klimaszewski sp. nov.

Subgeneric assignment. Aleochara.

Distribution. Brazil.

Aleochara solskyiana Likovský, 1984: 5. [Type locality: “Cayenne" (SOLSKy 1870)]. Note: Replacement name for A. humeralis Solsky, 1870, preoccupied by A. humeralis Gravenhorst, 1802. Aleochara humeralis Solsky, 1870: 257 (not Gravenhorst, 1802). [Type locality: "Cayenne" (Solsky 1870)].

Remarks. The above list of synonyms is from LikovskÝ (1984). Subgeneric assignment. Aleochara (Bernhauer \& SCHEERPeltz 1926).

Distribution. French Guiana (BLACKWELDER 1944).

Aleochara verecunda Sharp, 1876: 69. [Type locality: "Tapajos" (SHARP 1876)].

Subgeneric assignment. Aleochara (Bernhauer \& SCHEERPeltz 1926).
Distribution. Brazil (BLACKWELDER 1944).

\section{Subgenus Calochara Casey, 1906}

Type species: Calochara rubripennis Casey, 1906: 150. Fixed by CASEY (1906) by original designation and monotypy. For review of literature, see Gouix \& KLIMASZEwski (2007). For redescription of the subgenus, see KLIMASzewski (1984).

Aleochara andina Pace, 1987: 517. [Type locality: "Santiago, Puelma" (PACE 1987)].

Subgeneric assignment. Calochara (PACE 1987).

Distribution. Chile (PACE 1987).

Aleochara speculicollis Bernhauer, 1901: 371. [Type locality: "USA, Texas" (KLIMASZEwski 1984)].

Subgeneric assignment. Calochara (KLIMASzEWSKI 1984).

Distribution. Mexico; Nearctic region (NAvarrete-Heredia et al. 2002).

\section{Subgenus Coprochara Mulsant \& Rey, 1874}

Type species: Aleochara bilineata Gyllenhal, 1810: 436 Fixed by Fenyes (1918) in subsequent designation. For review of literature, see Gouix \& KLIMASZEwsKi (2007). For redescription of the subgenus, see KLIMASZEWSKI (1984).

Aleochara bilineata Gyllenhal, 1810: 436. [Type locality: "Switzerland, Unter-Engadin, Alp Urschein" (Maus 1998)].

Aleochara agilis Stephens, 1832: 154. [Type locality: "England" (Klimaszewski 1984)]

Aleochara immaculata Stephens, 1832: 154. [Type locality: "England" (Kuimaszewski 1984)].

Aleochara alpicola Heer, 1839: 317. [Type locality: "Switzerland, Alps near Unter Engadin" (Kurmaszewski 1984)].

Aleochara nigricornis Gredler, 1866: 464. [Type locality: “Austria, southern Tyrol" (KLIMASzEwsKi 1984)].

Aleochara anthomyiae Sprague, 1870: 370. [Type locality: "Massachusetts, Boston" (KLIMAszewski 1984)].

Baryodma ontarionis Casey, 1916: 71. [Type locality: “Canada, Ontario, Ottawa" (KLIMASZEWSKI 1984)].

Remarks. The above list of synonyms is modified from Maus (1998). Note: In Maus (1998) there are two other listed synonyms, Aleochara nitida Erichson, 1837: 358 (not Gravenhorst, 1802) and Aleochara bimaculata Burks, 1952: 379 (not Gravenhorst, 1802), which are only misidentifications by the respective authors.

Subgeneric assignment. Coprochara (Kumaszewski 1984).

Distribution. Guadeloupe; Nearctic, Palaearctic and Oriental regions (KLIMASzewsKi 1984; Maus 1998).

Aleochara bimaculata Gravenhorst, 1802: 187. [Type locality: "Pennsylvania" (KLIMAszewsKi 1984)].

Baryodma obsolescens Casey, 1906: 158. [Type locality: "USA, California" (KLIMASZEwsKi 1984)].

Baryodma recta Casey, 1906: 159. [Type locality: “USA, New Mexico" (Klimaszewski 1984)]. 
Baryodma deserticola Casey, 1906: 160. [Type locality: "USA, Arizona" (Klimaszewski 1984)].

Baryodma innocua Casey, 1906: 160. [Type locality: "USA, California" (KLIMASZEWSKI 1984)].

Remarks. The above list of synonyms is from KLIMAszewsKI (1984).

Subgeneric assignment. Coprochara (KLIMASzEwski 1984).

Distribution. Mexico, Guatemala, Colombia, Venezuela,

Ecuador, Brazil, Chile, Argentina, Haiti; Nearctic region (NavarRete-Heredia et al. 2002)

Aleochara composita (Casey, 1906: 164) (as Baryodma). [Type locality: "Mex D.F." (Maus 1998)].

Subgeneric assignment. Coprochara (MAus 1998).

Distribution. Mexico (Navarrete-Heredia et al. 2002).

Aleochara densissima Bernhauer, 1906: 345. [Type locality: "USA, California, Palm Springs" (Klimaszewski 1984)].

Baryodma densiventris Casey, 1906: 158 (not Bernhauer, 1906).

[Type locality: "USA, New Mexico" (KLIMAszewski 1984)].

Baryodma humboldti Casey, 1910: 108. [Type locality: "USA,

New Mexico" (Kuimaszewski 1984)]. Note: Replacement name for B. densiventris Casey, 1906, preoccupied by $A$. densiventris Bernhauer, 1906.

Remarks. The above list of synonyms is from KLIMASzEwsKI (1984).

Subgeneric assignment. Coprochara (KLIMASZEWSKI 1984).

Distribution. Mexico; Nearctic region (Navarrete-Heredia et al. 2002).

Aleochara mutare Blackwelder, 1944: 167. [Type locality: "Copiapo" (Solier 1849)]. Note: Replacement name for Mecorhopalus elongatus Solier, 1849, preoccupied by A. elongata Stephens, 1832 .

Mecorhopalus elongatus Solier, 1849: 348 (not Stephens, 1832).

[Type locality: "Copiapo" (Solier 1849)].

Remarks. The above list of synonyms is from Maus (1998). Subgeneric assignment. Coprochara (MAUs 1998).

Distribution. Chile (Maus 1998).

Aleochara notula Erichson, 1839: 167. [Type locality: "St. Thomas" (Maus 2001)].

Aleochara duplicata Erichson, 1839: 167. [Type locality: "Columbia" (Maus 2001)].

Baryodma nanella Casey, 1906: 160. [Type locality: "Brownsville, Texas" (Maus 2001)].

Baryodma nitidicollis Casey, 1906: 160 (not Solier, 1849). [Type locality: "Tex" (Maus 2001)].

Aleochara pernix Blackwelder, 1944: 167. [Type locality: "Tex" (Maus 2001)]. Note: Replacement name for B. nitidicollis Casey, 1906, preoccupied by A. nitidicollis Solier, 1849. Remarks. The above list of synonyms is from Maus (2001). Subgeneric assignment. Coprochara (Maus 2001).

Distribution. Mexico, Guatemala, El Salvador, Honduras, Nicaragua, Costa Rica, Panama, Colombia, Venezuela, Peru,
Bolivia, Brazil, Chile, Paraguay, Argentina, Cuba, Jamaica, Puerto Rico, Dominica; Nearctic region (Solsky 1872, PACE 1999, Maus 2001, Navarrete-Heredia et al. 2002).

Aleochara peschkeana Maus, 1999: 365. [Type locality: "Bogota, Columb." (Maus 1999)].

Subgeneric assignment. Coprochara (Maus 1999).

Distribution. Colombia (MAus 1999).

Aleochara pycnostichia Maus, 2000: 75. [Type locality: "Peru: Quebrada Verde bei Lima 100m" (Maus 2000)].

Subgeneric assignment. Coprochara (Maus 2000).

Distribution. Peru (Maus 2000).

Aleochara repetita Sharp, 1887: 776. [Type locality: "Panama, San Feliz; Amazons, Pará" (Sharp 1887)].

Subgeneric assignment. Coprochara. Note: Bernhauer \& Scheerpeltz (1926) cited this species within the subgenus Baryodma. Baryodma is a junior synonym of Coprochara (GouIx \& KLIMASzEWSKI 2007).

Distribution. Panama, Brazil (BlackWelder 1944).

Aleochara signaticollis Fairmaire \& Germain, 1861: 413. [Type locality: "Santiago" (Fairmaire \& Germain 1861)].

Subgeneric assignment. Coprochara (Maus 2001).

Distribution. Peru, Bolivia, Brazil, Chile, Argentina, Uruguay (Maus 2001).

Aleochara solieri Bernhauer \& Scheerpeltz, 1926: 794. [Type locality: "Carelmapú" (Solier 1849)]. Note: Replacement name for Mecorhopalus bipustulatus Solier, 1849, preoccupied by $A$. bipustulata Linné, 1760.

Mecorhopalus bipustulatus Solier, 1849: 348 (not Linné,

1760). [Type locality: "Carelmapú" (Solier 1849)].

Remarks. The above list of synonyms is from Maus (1998). Subgeneric assignment. Coprochara (Maus 1998).

Distribution. Chile (Maus 1998).

Aleochara sulcicollis Mannerheim, 1843: 225. [Type locality: "USA, Alaska, Sitka Is." (Kumaszewski 1984)].

Eucharina rugosa Casey, 1906: 166. [Type locality: "Canada, British Columbia, Queen Charlotte Islands" (KLIMASzEwSKI 1984)].

Eucharina tibialis Casey, 1906: 166. [Type locality: "USA, California" (Klimaszewski 1984)].

Eucharina cylindrella Casey, 1906: 167. [Type locality: "USA, California, San Diego." (KlimaszewsKi 1984)].

Eucharina debilicornis Casey, 1906: 167. [Type locality: "USA, California" (KLIMASzewski 1984)].

Remarks. The above list of synonyms is from KLIMASzEwskI (1984).

Subgeneric assignment. Coprochara (KLIMASzewsKi 1984).

Distribution. Mexico, Chile; Nearctic region (NavarreteHEREDIA et al. 2002).

Aleochara trachynoptera Maus, 2000: 72.[Type locality: "Brasil S. Paulo, Umgbg. v. Ribeirão Preto" (Maus 2000)]. 
Subgeneric assignment. Coprochara (Maus 2000).

Distribution. Colombia, Brazil (Maus 2000).

Aleochara verna Say, 1833: 58. [Type locality: "USA, Missouri" (KLimaszewski 1984)].

Aleochara bimaculata var. alticola Sharp, 1883: 148. [Type locality: "Guatemala, Totanicapan 8500 to $10,500 \mathrm{ft}^{\text {" }}$ (Klimaszewski 1984)].

Baryodma minuta Casey, 1906: 161. [Type locality: "California (San Francisco to Pomona) and Iowa" (CASEy 1906)].

Baryodma pumilio Casey, 1911: 6 (not Gravenhorst, 1802). [Type locality: "Iowa (Cedar Rapids)" (CASEY 1911)].

Baryodma tolerata Casey, 1911: 6. [Type locality: "California (Dunsmuir)" (CASEY 1911)].

Aleochara tecumsehi Muona, 1977: 16. [Type locality: "Iowa (Cedar Rapids)" (CASEy 1911)]. Note: Replacement name for A. pumilio (Casey, 1911) preoccupied by A. pumilio Gravenhorst, 1802 (Muona 1977).

Aleochara cedari Likovský, 1984: 8. [Type locality: "Iowa (Cedar Rapids)" (LikovskÝ 1984)]. Note: Replacement name for A. pumilio (Casey, 1911) preoccupied by A. pumilio Gravenhorst, 1802 (Likovskŕ 1984). The preoccupied name had already been replaced by Muona (1977).

Aleochara tanumi Likovský, 1984: 8. [Type locality: "California (San Francisco to Pomona) and Iowa" (Likovský 1984)]. Note: Replacement name for A. minuta (Casey, 1906) preoccupied by A. minuta Gravenhorst, 1806.

Remarks. The above list of synonyms is from NAVARRETEHeredia et al. (2002).

Subgeneric assignment. Coprochara (KLIMAszewsKi 1984).

Distribution. Mexico, Venezuela; Nearctic, Palaearctic, Oriental, Pacific regions (Navarrete-Heredia et al. 2002).

\section{Subgenus Emplenota Casey, 1884}

Type species: Emplenota maritima Casey, 1884: 17. Fixed by CASEy (1884) by original designation and monotypy. For review of literature, see Gouix \& Klimaszewski (2007). For redescription of the subgenus, see KLIMASZEWSKI (1984).

Aleochara litoralis (Mäklin, 1853: 182) (as Homalota). [Type locality: "USA, Kodjak" (KLIMASZEwSKi 1984)].

Emplenota maritima Casey, 1884: 17. [Type locality: “USA, New Jersey" (KLIMAszewski 1984)].

Polistoma arenaria Casey, 1894: 289. [Type locality: “USA, California" (KLIMASzewski 1984)].

Emplenota quadrifer Casey, 1906: 175. [Type locality: “USA, California, Redondo" (KLIMAszewski 1984)].

Emplenota trilimbata Casey, 1906: 175. [Type locality: “USA, California" (KLIMAszewski 1984)].

Emplenota longiceps Casey, 1911: 9. [Type locality: "Canada, British Columbia, Metlakatla" (KLIMAszewski 1984)].

Remarks. The above list of synonyms is from KLIMASzEWSKI (1984).

Subgeneric assignment. Emplenota (KLIMASzEwsKi 1984).
Distribution. Mexico; Nearctic region (NAVARRETe-HerediA et al. 2002).

Aleochara pacifica (Casey, 1894: 290) (as Polistoma). [Type locality: "USA, California, Sta. Barbara" (KLIMAszewski 1984)].

Subgeneric assignment. Emplenota (KLIMASZEwsKi 1984).

Distribution. Mexico; Nearctic region (NAVArRETe-Heredia et al. 2002).

\section{Subgenus Maseochara Sharp, 1883}

Type species: Maseochara opacella Sharp, 1883: 154. Fixed by Fenyes (1918) by subsequent designation. For review of literature, see KLIMASzEWSKI (1984). For redescription of the subgenus, see KLIMASZEWSKI (1984).

Aleochara depressa (Sharp, 1883: 155) (as Maseochara). [Type locality: "Guatemala" (KLIMASzewski 1984)].

Maseochara puberula Casey, 1894: 284. [Type locality: "USA, Arizona" (KLIMASZEwSKi 1984)].

Maseochara basalis Casey, 1906: 171. [Type locality: "USA, California, Arrowhead" (Klimaszewski 1984)].

Maseochara musta Casey, 1911: 7. [Type locality: "USA, Arizona" (KLIMASZEWSKi 1984)].

Remarks. The above list of synonyms is from KLIMASZEWSKI (1984).

Subgeneric assignment. Maseochara (KLIMASZEwSKI 1984).

Distribution. Mexico, Guatemala; Nearctic region (Navarrete-Heredia et al. 2002).

Aleochara gracilis (Sharp, 1883: 155) (as Maseochara). [Type locality: "Mexico, Guanajuato" (Kuimaszewski 1989)].

Subgeneric assignment. Maseochara (NAvarrete-Heredia et al. 2002).

Distribution. Mexico (Navarrete-Heredia et al. 2002).

Aleochara missionis Klimaszewski \& Maus, 1999: 219. [Type locality: "Republic Argentina, Misiones" (Kumaszewski \& Maus 1999)]. Note: Replacement name for Maseochara argentina Bernhauer, 1927, preoccupied by A. argentina Bernhauer, 1908.

Maseochara argentina Bernhauer, 1927: 264 (not Bernhauer, 1908). [Type locality: "Republic Argentina, Misiones" (Klimaszewski \& Maus 1999)].

Remarks. The above list of synonyms is from KLIMAszewski \& MAUS (1999).

Subgeneric assignment. Maseochara (KLIMASZEwSKI \& MAUS 1999).

Distribution. Argentina (Klimaszewski \& Maus 1999).

Aleochara newtoni Caron, Mise \& Klimaszewski, nom. nov. [Type locality: "Guatemala, near the city" (KLIMAszewski 1989)].

Maseochara duplicata Sharp, 1883: 156 (not Erichson, 1839). [Type locality: "Guatemala, near the city" (KLImAszewski 1989)]. Note: This is a junior secondary homonym of $A$. 
duplicata Erichson, 1839, which is still congeneric (synonym of $A$. notula Erichson, 1839, above) (Alfred F. Newton, from FMNH, pers. comm.).

Remarks. We follow Article 59.1 and 60.3 of the International Code of Zoological Nomenclature (ICZN), which requires that, respectively, "a junior secondary homonym must be treated as invalid by anyone who considers that the two species-group taxa in question are congeneric" and "If the rejected junior homonym has no known available and potentially valid synonym it must be replaced by a new substitute name, with its own author and date" (ICZN 1999).

Etymology of the new name. In honor of Dr. Alfred F. Newton, from FMNH, for his valuable information on nomenclatural problems of Aleochara species from the Neotropical region.

Subgeneric assignment. Maseochara (NAVARRETe-Heredia et al. 2002).

Distribution. Mexico, Guatemala, Panama (NavarreteHeredia et al. 2002).

Aleochara opacella (Sharp, 1883: 154) (as Maseochara). [Type locality: "Mexico, Tehuacan" (KLimaszewski 1984)].

Maseochara hogei Sharp, 1887: 777. [Type locality: "Mexico" (KLimaszewski 1984)].

Maseochara decipiens Casey, 1906: 169. [Type locality: "USA, Arizona" (KuImaszewski 1984)].

Remarks. The above list of synonyms from KuIMASzewsKI (1984).

Subgeneric assignment. Maseochara (KLIMASZEWSKI 1984).

Distribution. Mexico; Nearctic region (NavarRete-Heredia et al. 2002).

Aleochara semivelutina Solsky, 1868: 120. [Type locality: "Mexique et probablement de l'Etat d'Oaxaca" (Solsky 1868).

Note: Solsky (1868) gives this information at the beginning of the paper for all species described in that paper].

Subgeneric assignment. Maseochara (NAVARRETe-Heredia et al. 2002).

Distribution. Mexico; Nearctic region (NAvarrete-Heredia et al. 2002).

Aleochara valida LeConte, 1858: 16. [Type locality: “USA, California, San Diego" (KLimaszewski 1984)].

Maseochara robusta Sharp, 1883: 155. [Type locality: "Mexico, Tehuacan" (KLImaszewski 1984)].

Maseochara californica Casey, 1885: 309. [Type locality: "USA, California" (KLIMASZEWski 1984)].

Maseochara ponderosa Casey, 1906: 170. [Type locality: “USA, Arizona" (Kumaszewski 1984)].

Maseochara ruficauda Casey, 1906: 170. [Type locality: "USA, Arizona" (KuImaszewski 1984)].

Remarks. The above list of synonyms is from KLIMASZEWSKI (1984).

Subgeneric assignment. Maseochara (KLIMASzEwsKi 1984).

Distribution. Mexico; Nearctic region (Navarrete-Heredia et al. 2002).

\section{Subgenus Xenochara Mulsant \& Rey, 1874}

Type species: Aleochara decorata Aubé, 1850: 311. Fixed by Mulsant \& Rey (1874) by monotypy. For review of literature, see Gouix \& Klimaszewski (2007). For redescription of the subgenus, see KLIMASZEWSKI (1984).

Aleochara alajuela Klimaszewski \& Ashe, 1992: 405. [Type locality: "Costa Rica: Alajuela Peñas Blancas, 1420m" (Kuimaszewski \& Ashe, 1992)].

Subgeneric assignment. Xenochara (KLIMAszewski \& Ashe, 1992).

Distribution. Costa Rica (KlimaszewsKi \& Ashe, 1992).

Aleochara aliipennis Bernhauer, 1927: 264. [Type locality: "Argentina: Masao Catamarca" (Klimaszewski \& Maus 1999)].

Subgeneric assignment. Xenochara (KLimaszewski \& Maus 1999).

Distribution. Chile, Argentina (KLIMAszewski \& Maus 1999).

Aleochara angusticeps Sharp, 1883: 153. [Type locality: "Guatemala, Paso Antonio 400 feet" (KuIMaszewsKi 1989)].

Subgeneric assignment. Xenochara (KLIMASzEwski 1989).

Distribution. Guatemala (KLIMASzEwski 1989). Note: There is one doubtful record from the USA (Tennessee) (KLIMASzEwskI 1989).

Aleochara argentina Bernhauer, 1908: 371. [Type locality: in original description: "Argentinien: Chaunar-Region" (BERNHAUER 1908), and on original label: "Brazil Chaunar-Region" (Klimaszewski \& Maus 1999, based in lectotype designated)].

Subgeneric assignment. Xenochara (KLIMASzewski \& Maus 1999).

Distribution. Brazil, Argentina (Klimaszewski \& Maus 1999; Alfred F. Newton, from FMNH, pers. comm.).

Aleochara brooksi Klimaszewski \& Ashe, 1992: 402. [Type locality: "Costa Rica: Alajuela Peñas Blancas, 870m" (Kuimaszewski \& Ashe 1992)].

Subgeneric assignment. Xenochara (KLIMASzewski \& Ashe 1992).

Distribution. Costa Rica (Klimaszewski \& Ashe 1992).

Aleochara bruchiana Bernhauer, 1927: 263. [Type locality: "Republic Argentina, Prov. San Juan" (Klimaszewski \& Maus 1999)]. 1999).

Subgeneric assignment. Xenochara (KLIMASzewski \& Maus

Distribution. Argentina (Klimaszewski \& Maus 1999).

Aleochara costarica Klimaszewski \& Ashe, 1992: 403. [Type locality: "Costa Rica: Alajuela Peñas Blancas, 1420m" (Kuimaszewski \& Ashe 1992)].

Subgeneric assignment. Xenochara (KLIMASzewski \& Ashe 1992).

Distribution. Costa Rica (Klimaszewski \& Ashe 1992). 
Aleochara divergens Pace, 1990: 172. [Type locality: "Perù, dep. Cuzco, Cosnipata Ebene, 1000m" (PACE 1990)]. Subgeneric assignment. Xenochara (PACE 1990). Distribution. Peru (PACE 1990).

Aleochara felixiana Klimaszewski, 1989: 2. [Type locality: "Panama, San Felix" (KLIMASzewsKi 1989)].

Subgeneric assignment. Xenochara (KLIMASZEwSKI 1989). Distribution. Panama (KLIMASzEWSKI 1989).

Aleochara festiva Pace, 1990: 172. [Type locality: "Brasilien, Nova Teutonia" (PACE 1990)].

Subgeneric assignment. Xenochara (PACE 1990).

Distribution. Brazil (PACE 1990).

Aleochara forsteri Scheerpeltz, 1960: 136. [Type locality: "Bolivien, Cochabamba, 3650 m, Lagunas de Vacas" (Scheerpeltz 1960)].

Subgeneric assignment. Xenochara. Note: Scheerpeltz (1960) cited this species within subgenus Polychara. Polychara is a junior synonym of Xenochara (Gouix \& KLIMASzEwski 2007). Distribution. Bolivia (Scheerpeltz 1960).

Aleochara funestior Sharp, 1883: 151. [Type locality: "Panama, Volcan de Chiriqui, 3000 to $4000 \mathrm{ft}$ " (KuIMaszewski 1989)].

Subgeneric assignment. Xenochara (KLIMAszewski 1989).

Distribution. Guatemala, Panama, Colombia (Newton et al. 2005).

Aleochara glabra Bernhauer, 1921: 179. [Type locality: "Republic Argentina, Prov. Jujuy" (Kumaszewski \& Maus 1999)]. 1999).

Subgeneric assignment. Xenochara (KLIMAszewski \& Maus

Distribution. Argentina (Klimaszewski \& Maus 1999).

Aleochara lacertina Sharp, 1883: 148. [Type locality: "Mexico, Jalapa" (Klimaszewski 1984)].

Baryodma idonea Casey, 1906: 157. [Type locality: “USA, New Jersey" (KuIMaszewski 1984)].

Baryodma imbricata Casey, 1906: 157. [Type locality: “USA, California" (KLIMASzewsKi 1984)].

Baryodma salicola Casey, 1906: 157. [Type locality: “USA, California, Colorado Desert at Salton" (Klimaszewski 1984)].

Aleochara (Isochara) densiventris Bernhauer, 1906: 346. [Type locality: "USA, California, Pasadena" (KLIMASzEwski 1984)]. Remarks. The above list of synonyms is from NAVARRETEHeredia et al. (2002).

Subgeneric assignment. Xenochara (KLIMASzEwsKi 1984).

Distribution. Mexico; Nearctic region (NAvarrete-Heredia et al. 2002).

Aleochara lacustris Bernhauer, 1908: 372. [Type locality: "Peru: Titicaca-See" (BERnhauer 1908)]. 1999).

Subgeneric assignment. Xenochara (KLIMASzewski \& MaUs

Distribution. Peru (Kumaszewski \& Maus 1999).
Aleochara lescheni Klimaszewski \& Ashe, 1992: 401. [Type locality: "Costa Rica: Alajuela Peñas Blancas, 800m" (KLIMAszewski \& Ashe 1992)].

Subgeneric assignment. Xenochara (KLimaszewski \& Ashe 1992).

Distribution. Costa Rica (KLIMASzewski \& Ashe 1992).

Aleochara mexicana Sharp, 1883: 149. [Type locality: "Mexico, Juquila" (KLIMASZEWSKI 1989)].

Aleochara miradoris Sharp, 1883: 152. [Type locality: "Mexico, Mirador" (Klimaszewski 1989)].

Remarks. The above list of synonyms is from NAVARrETEHEREDia et al. (2002).

Subgeneric assignment. Xenochara (Navarrete-Heredia et al. 2002).

Distribution. Mexico, Guatemala, Costa Rica (NAvarreteHeredia et al. 2002).

Aleochara monteverde Klimaszewski \& Ashe, 1992: 404. [Type locality: "Costa Rica: Alajuela Peñas Blancas, 1190m" (Klimaszewski \& Ashe 1992)].

Subgeneric assignment. Xenochara (KLimaszewski \& Ashe 1992).

Distribution. Costa Rica (KLIMASzewsKi \& Ashe 1992).

Aleochara opacula Bernhauer, 1901: 369. [Type locality:

"Amazonas" (KuIMaszewski \& Maus 1999)].

Subgeneric assignment. Xenochara (KLIMASzewski \& Maus 1999).

Distribution. Brazil (KLIMASzewsKi \& Maus 1999).

Aleochara otongo Klimaszewski, 1990: 172. [Type locality: "Mexico, Chiapas, Lacaohatan, La Victoria, Alt. 430m" (KLIMASZEWSKI 1990)].

Subgeneric assignment. Xenochara (KLIMASzEwSKI 1990). Distribution. Mexico (Navarrete-Heredia et al. 2002).

Aleochara oxypodia Sharp, 1883: 150. [Type locality: "Panama, Volcan de Chiriqui, 2500 to 4000 feet" (KLimaszewski 1989)]. Subgeneric assignment. Xenochara (KuIMASzEwski 1989).

Distribution. Mexico, Guatemala, Panama (NAvarreteHeredia et al. 2002).

Aleochara puberula Klug, 1832: 139. [Type locality: "Madagaskar" (KuIMASZEWSKI 1984)].

Aleochara deserta Erichson, 1839: 173. [Type locality: “Arabia desert" (KLIMASzewski 1984)].

Aleochara vaga Erichson, 1839: 172. [Type locality: "Promont. bon. Spei, Dom. Bergius" (Klimaszewski 1984)].

Aleochara decorata Aubé, 1850: 311. [Type locality: "France, probably near Paris" (KumaszewsKi 1984)].

Aleochara armitagei Wollaston, 1854: 559. [Type locality: "Madeira" (KLimaszewski 1984)].

Oxypoda sanguinolenta Motschulsky, 1858: 241. [Type locality: "Egypt, Alexandria" (Kumaszewski 1984)].

Oxypoda brunescens Motschulsky, 1858: 243. [Type locality: "East Indies" (Klimaszewski 1984)]. 
Aleochara dubia Fauvel, 1863: 428. [Type locality: "Cuba" (KLIMASZEWSKi 1984)].

Oxypoda analis MacLeay, 1873: 135. [Type locality: "Australia, Gayndah, near Burnett River" (KLIMAszewski 1984)].

Baryodma bipartita Casey, 1894: 287. [Type locality: "USA, Texas, Galveston" (Kumaszewski 1984)].

Aleochara major Eichelbaum, 1912: 176. [Type locality: "Africa, Parehberg, 1600 m" (KuIMaszewski 1984)].

Remarks. The above list of synonyms is from KLIMAszewsKI (1984).

Subgeneric assignment. Xenochara (KLIMASzEwski 1984).

Distribution. South America, Cuba, Guadeloupe; Nearctic, Palaearctic, Afrotropical, Australian regions (KLIMAszewski 1984, Navarrete-Heredia et al. 2002).

Aleochara punae Pace, 1990: 170. [Type locality: "Bolivia, Yongas de La Paz, 1000 m" (PaCe 1990)].

Subgeneric assignment. Xenochara (PACE 1990).

Distribution. Bolivia (PACE 1990).

Aleochara quadrata Sharp, 1883: 149. [Type locality: "Mexico" (KLIMASZEWSKi 1984)].

Baryodma uvidula Casey, 1906: 153. [Type locality: "USA, California" (KuIMASZEwski 1984)].

Aleochara sparsicollis Bernhauer, 1906: 347. [Type locality: "USA, California, Monterey Co." (KuIMASZEwSKi 1984)].

Remarks. The above list of synonyms is from KLIMASzEWSKI (1984).

Subgeneric assignment. Xenochara (KLIMASzewski 1984).

Distribution. Mexico; Nearctic region (Navarrete-Heredia et al. 2002).

Aleochara richteri Bernhauer, 1912: 82. [Type locality: "Comodoro, Rivadavia, Gobernacion, Santa Cruz" (KLIMASzewski \& Maus 1999)].

Aleochara wittei Bernhauer, 1921: 178. [Type locality: "Republic Argentina, Gob. Santa Cruz, 190" (KLIMAszewski \& Maus 1999)].

Remarks. The above list of synonyms is from KLIMAszewskI $\&$ Maus (1999). 1999).

Subgeneric assignment. Xenochara (KLIMAszewsKi \& Maus

Distribution. Argentina (Kumaszewski \& Maus 1999).

Aleochara sallaei Sharp, 1883: 152. [Type locality: "Mexico, Guanajuato" (KLIMASZEwski 1984)].

Aleochara torquata Sharp, 1883: 153. [Type locality: "Panama, Volcan de Chiriquí 4000 to $6000 \mathrm{ft}$ " (KLIMASzewski 1984)].

Remarks. The above list of synonyms is from KLIMAszewsKI (1984).

Subgeneric assignment. Xenochara (KLIMASzEwsKi 1984).

Distribution. Mexico, Guatemala, Panama; Nearctic region (Navarrete-Heredia et al. 2002).

Aleochara simulatrix Sharp, 1883: 151. [Type locality: "Guatemala, El Tumbador, $2500 \mathrm{ft}$ " (KLIMAszewski 1989)].
Subgeneric assignment. Xenochara (KLIMASzEwsKI 1989). Distribution. Guatemala, Panama (Sharp 1883, 1887).

Aleochara taeniata Erichson, 1839: 165. [Type locality: "St. Jean" (Klimaszewski 1984)].

Oxypoda lapidicola Sahlberg, 1844: 517. [Type locality: "Brazil, Rio de Janeiro" (KLImaszewski 1984)].

Remarks. The above list of synonyms is from KLIMASzewskI (1984).

Subgeneric assignment. Xenochara (KLIMASzewsKi 1984).

Distribution. Mexico, Guatemala, Costa Rica, Panama, Colombia, French Guiana, Peru, Brazil, West Indies (Fauvel 1901; Klimaszewski 1984; Navarrete-Heredia et al. 2002).

Aleochara verberans Erichson, 1839: 164. [Type locality: "Colombia" (KLIMASZEWSKi \& GéNIER 1986)].

Subgeneric assignment. Xenochara (KLIMAszewski et al. 1987).

Distribution. Mexico, Colombia, Venezuela, Brazil, Paraguay, Argentina, Uruguay, Cuba (Navarrete-Heredia et al. 2002).

Aleochara weiseri Bernhauer, 1921: 178. [Type locality: "Republic Argentina, Prov. Jujuy" (KLimaszewski \& Maus 1999)].

Subgeneric assignment. Xenochara (KLIMAszewski \& Maus 1999).

Distribution. Argentina (KLIMASzewski \& Maus 1999).

\section{Incertae Sedis}

Aleochara nitidicollis Solier, 1849: 352. [Type locality: "Illapel" (SOLIER 1849)].

Subgeneric assignment. Unknown. Note: Bernhauer \& SCheerpeltz (1926) cited this species as "species dubiae" in Aleochara. Distribution. Chile (BLACKWELDER 1944).

\section{ACKNOWLEDGMENTS}

We thank Alfred F. Newton (FMNH) for valuable nomenclatoral information on Aleochara species from the Neotropical region. The following people contributed to the improvement of the manuscript by their valuable comments: Cibele S. RibeiroCosta, Lucia M. de Almeida, Germano H. Rosado-Neto, Angélico Asenjo, all from UFPR, and two anonymous reviewers. We thank the Biological Collection Network of Paraná (Taxonline, UFPR) for providing the photographs; and the Conselho Nacional de Desenvolvimento Científico e Tecnológico and the Coordenação de Aperfeiçoamento de Pessoal de Nível Superior for the scholarships to the first and second author, respectively. We also appreciate the useful comments made by Pamela Cheers, Laurentian Forestry Centre. This is contribution number 1749 of the Departamento de Zoologia, Universidade Federal do Paraná.

\section{LITERATURE CITED}

Aubé, C. 1850. Description de quelques insectes coléoptères appartenant à l'Europe et à l'Algérie. Annales de la Société Entomologique de France 8: 299-346. 
Bernhauer, M. 1901. Neue exotische Arten der Gattung Aleochara Grav. Stettiner Entomologische Zeitung 62: 366-373.

Bernhauer, M. 1904. Neue exotische Staphyliniden. Stettiner Entomologische Zeitung 65: 217-242.

Bernhauer, M. 1906. Neue Aleocharinen aus Nord-America. Deutsche Entomologische Zeitschrift 1906: 337-348.

Bernhauer, M. 1908. Beitrag zur Staphylinidenfauna von Südamerika. Archiv für Naturgeschichte 74: 283-372.

Bernhauer, M. 1912. Zur Staphylinidenfauna von Südamerika. Wiener Entomologische Zeitung 31: 68-82.

Bernhauer, M. 1921. Neue Staphyliniden aus Südamerika, besonders aus Argentinien. Wiener Entomologische Zeitung 38: 101-108, 169-179.

Bernhauer, M. 1927. Zur Staphylinidenfauna Südamericas, insbesondere Argentiniens. Archiv für Naturgeschichte (1925) (A) 91: 229-264.

Bernhauer, M. \& O. Scheerpeltz. 1926. Staphylinidae VI. In: S. SCHENKLING (Ed.). Coleopterorum Catalogus. Berlin, Junk, p.499-988.

BLACKWELDER, R.E. 1936. Morphology of the coleopterous family Staphylinidae. Smithsonian Miscellaneous Collections 94: 1-102.

BlackWELDER, R.E. 1944. Checklist of the coleopterous insects of Mexico, Central America, the West Indies, and South America. Part 1. United States National Museum Bulletin 185: XII+1-188.

BornemisszA, G.F. 1957. An analysis of arthropod succession in carrion and the effect of its decomposition on the soil fauna. Australian Journal of Zoology 5: 1-12.

Bromand, B. 1980. Investigations on the biological control of the cabbage root fly (Hylemya brassicae) with Aleochara bilineata. Bulletin de l'Organisation Internationale de Lutte Biologique, Section Régionale Ouest-Paléarctique 3: 49-62.

BurKs, B.D. 1952. Other controls. Insects, enemies of insects, p. 373-380. In: Insects. The yearbook of agriculture. Washington, U.S. Gov. Printing Office, 780p.

Cameron, M. 1923. Descriptions of new species of Staphylinidae from the West Indies. Part II. The Annals and Magazine of Natural History 11: 363-400.

Casey, T.L. 1884. Contributions to the descriptive and systematic coleopterology of North America. Part I. Philadelphia, Collins Printing House, 60p.

Casey, T.L. 1885. New genera and species of Californian Coleoptera. Bulletin of the California Academy of Sciences 1: 283-336.

Casey, T.L. 1894. Coleopterological notices V. Annals of the New York Academy of Sciences 7: 281-606.

Casey, T.L. 1906. Observations on the staphylinid groups Aleocharinae and Xantholinini chiefly of America. Transactions of the Academy of Science of St. Louis 16: 125-434.

Casey, T.L. 1910. Synonymie and other notes on Coleoptera. Canadian Entomologist 42: 105-113.
Casey, T. L. 1911. New American species of Aleocharinae and Myllaeninae. Memoirs on the Coleoptera 2. Lancaster, Pennsylvania, New Era Printing, 245p.

Casey, T.L. 1916. A new species of Baryodma. Canadian Entomologist 48: 70-71.

Duvivier, A. 1883. Énumération des Staphylinides, décrits depuis la publication du catalogue de M. M. Gemminger et de Harold. Annales de la Société Entomologique de Belgique 27: 91-215.

Eichelbaum, F. 1912. Die von Herrn Dr. Christoph Schröder in den Parehbergen und auf dem Kilimandjaro im Januar 1906 gesammelten Staphylinidae (darunter zwei neue Philonthusarten). Berliner Entomologische Zeitschrift 56 (1911): 173-176.

ERICHSON, W.F. 1837. Die Käfer der Mark Brandenburg 1, Berlin, $384 \mathrm{p}$.

ERICHSON, W.F. 1839. Genera et species Staphylinorum insectorum coleopterorum familiae 1. Berlin, F.H. Morin, 400p.

FABRICIUS, J.C. 1801. Systema eleutheratorum secundum ordines, genera, species: adiectis synonymis, locis, observationibus, descriptionibus. Kiliae, Bibliopolii Academici Novi, vol. 2, 687p.

FAirmaire, L. \& P. Germain. 1861. Révision des Coléoptères du Chili. Annales de la Société Entomologique de France 1: 405-456.

Fauvel, A. 1863. [New species]. In: A. Chevrolat \& A. Fauvel. Coléoptères de l'Ile de Cuba. Notes, synonymiques et descriptions d'espèces nouvelles. Annales de la Société Entomologique de France 3: 427-446.

Fauvel, A. 1866. Faune du Chile. Insectes Coléoptères. Staphylinides. Bulletin de la Société Linnéenne de Normandie 10: 250-353.

Fauvel, A. 1901. Voyage de M. le Dr. Ed. Bugnion au Venezuela, en Colombie et aux Antilles. Revue d'Entomologie 20: 6991.

Fenyes, A. 1918. Coleoptera. Fam. Staphylinidae, subfam. Aleocharinae. Genera Insectorum 173: 1-110.

Fleming, J. 1821. Insecta, p. 41-56. In: Supplement to the fourth, fifth, and sixth editions of the Encyclopedia Britannica, with preliminary dissertations on the history of the sciences. Edinburgh, Archibald Constable and Company, vol. 5.

Fourcroy, A.F. DE. 1785. Entomologia Parisiensis; sive catalogus insectorum quae in agro parisiensi reperiuntur; secundum methodum Geoffraeanum in sectiones, genera et species distributus; cui addita sunt nomina trivalia et fere trecentae novae species. Parisiis, Aedibus Serpentineis, Pars prima, VIII+231p.

Goeze, J.A.E. 1777. Entomologische Beyträge zu des Ritter Linné zwölften Ausgabe des Natursystems 1. Leipzig, Weidmanns Erben und Reich, XVI+736p.

Gouix, N. \& J. KLimaszewski. 2007. Catalogue of Aleocharinae rove beetles of Canada and Alaska (Coleoptera, Staphyli- 
nidae, Aleocharinae). Sofia, Pensoft Publishers, Pensoft Series Faunistica 65, 165p.

Gravenhorst, J.L.C. 1802. Coleoptera Microptera Brunsvicensia nec non exoticorum quotquot exstant in collectionibus entomologorum Brunsvicensium in genera familias et species distribuit. Brunsuigae, Carolus Reichard, LXVI+206p.

Gravenhorst, J.L.C. 1806. Monographia Coleopterorum Micropterorum. Gottingae, Henricus Dieterich, 248p.

Gredler, P.V.M. 1866. Die Käfer von Tirol nach ihrer horizontalen und vertikalen Verbreitung II. Bozen, G. Ferrari, p. 235-491.

Gyllenhal, L. 1810. Insecta Suecica descripta. Classis I. Coleoptera sive Eleuterata 1. Scaris, F. J. Leverentz, $\mathrm{XX}+660 \mathrm{p}$.

HeEr, O. 1839. Fauna Coleopterorum Helvetica 1. Turici, Orelii, Fuesslini et Sociorum, p. 145-360.

Herman, L.H. 2001. Catalog of the Staphylinidae (Insecta: Coleoptera) 1758 to the end of the second millennium, parts I-VII. Bulletin of the American Museum of Natural History 167: 1-4218.

IczN. 1999. International Code of Zoological Nomenclature, fourth edition [on line]. The International Trust for Zoological Nomenclature, available online at http:// www.iczn.org/iczn/index.jsp [Accessed: 13/III/2008].

JoNASSON, T. 1990. Mustard meal mulching: a biological method for cabbage root fly control. Nordisk Jordbrugsforskning 72: 453.

Jonasson, T. 1994. Parasitoids of Delia root flies in brassica vegetable crops: coexistance and niche separation in two Aleochara species (Coleoptera: Staphylinidae). Norwegian Journal of Agricultural Sciences suppl. 16: 379-386.

Jones, C.M. 1967. Aleochara tristis, a natural enemy of Face Fly. I. Introduction and laboratory rearing. Journal of Economic Entomology 60: 816-817.

Klimaszewski, J. 1984. A revision of the genus Aleochara Gravenhorst of America North of Mexico (Coleoptera: Staphylinidae: Aleocharinae). Memoirs of the Entomological Society of Canada 129: 1-211.

KLIMASZEWSKI, J. 1989. A review of Sharp's types of Aleochara from Latin America (Coleoptera: Staphylinidae). Entomologica Scandinavica 20: 1-14.

Klimaszewski, J. 1990. Two new species and new records of Aleochara from Latin America (Coleoptera: Staphylinidae). Annals of the Transvaal Museum 35: 171-176.

Klimaszewski, J. \& J.S. Ashe. 1992. Aleochara Gravenhorst (Coleoptera: Staphylinidae: Aleocharinae) of the Monteverde Region of Costa Rica. Annals of the Transvaal Museum 35: 399410.

Klimaszewski, J. \& F. Génier. 1986. Lectotype designation and redescription of Aleochara verberans (Coleoptera: Staphylinidae). Entomological News 97: 115-118.

Klimaszewski, J. \& R.E. JANSEn. 1993. Systematics, biology and distribution of Aleochara Gravenhorst from Southern Africa.
Part I: subgenus Xenochara Mulsant \& Rey (Coleoptera: Staphylinidae). Annals of the Transvaal Museum 36: 53 107.

Klimaszewski, J. \& C. Maus. 1999. Review of Bernhauer's types of Aleochara from South America (Coleoptera: Staphylinidae: Aleocharinae). Zoological Studies 38: 207-221.

Klimaszewski, J.; J.H. Frank \& S.B. Peck. 1990. Two new species and a key to the adults of Aleochara of Florida (Coleoptera: Staphylinidae). Florida Entomologist 73: 177-185.

Klimaszewski, J.; F. GÉnier \& M. Uhlig. 1987. Review of Erichson's types of Aleochara from Mexico, West Indies and South America. Florida Entomologist 70: 249-259.

KLUG, J.C.F. 1832. Bericht über eine auf Madagascar veranstaltete Sammlung von Insecten aus der Ordnung Coleoptera. Abhandlungen der Königlichen Akademie der Wissenschaften zu Berlin 1832: 91-223.

LAPORTE, F.L. (Castelnau). 1835. Études entomologiques, ou description d'insectes nouveaux, et observations sur leur synonymie. Paris, Méquignon-Marvis, 159p.

LeConte, J.L. 1858. Catalogue of Coleoptera of the regions adjacent to the boundary line between the United States and Mexico. Proceedings of the Academy of Natural Sciences of Philadelphia 4: 9-42.

LıKovskŕ, Z. 1984. Über die nomenklatur der Aleocharinen (Coleoptera, Staphylinidae). Annotationes Zoologicae et Botanicae 160: 1-8.

LINNÉ, C. V. 1758. Systema naturae, per regna tria naturae, secundum Classes, Ordines, Genera, Species, cum characteribus, differentiis, synonymis, locis. Holmiae, Laurentii Salvii, $10^{\text {th }}$ ed., vol. $1,824 \mathrm{p}$.

LINNÉ, C. V. 1760. Fauna suecica sistens animalia sueciae regni; Mammalia, Aves, Amphibia, Pisces, Insecta, Vermes. Distributa per classes et ordines, genera et species, cum differentiis specierum, synonymis auctorum, nominibus incolarum, locis natalium descriptionibus insectorum. Stockholmiae, Laurentii Salvii, $2^{\text {nd }}$ ed., 49 unnumbered pages $+578 \mathrm{p}$.

LyNCH, F.A. 1884. Los estafilinos de Buenos Aires. Boletin de la Academia Nacional de Ciencias, Córdoba 7: 5-392.

MacLeay, W.J. 1873. Notes on a collection of insects from Gayndah. The Transactions of the Entomological Society of New South Wales 2: 79-205.

MäKLIN, F.G. 1853. [New species and notes]. In: C.V. Mannerheim, Dritter Nachtrag zur Kaefer-Fauna der Nord-Amerikanischen Laender des Russischen Rieches. Bulletin de la Société Impériale des Naturalistes de Moscou 26: 95-273.

Mannerheim, C.G.V. 1843. Beitrag zur Kaefer-Fauna der Aleutischen Inseln, der Insel Sitkha und Neu-Californiens. Bulletin de la Société Impériale des Naturalistes de Moscou 16: $175-314$.

Maus, C. 1998. Taxonomical contributions to the subgenus Coprochara Mulsant \& Rey, 1874 of the genus Aleochara Gravenhorst, 1802 (Coleoptera: Staphylinidae). Koleoptero- 
logische Rundschau 68: 81-100.

Maus, C. 1999. Four new species of the genus Aleochara Gravenhorst, 1802, subgenus Coprochara Mulsant \& Rey, 1874 (Coleoptera: Staphylinidae). Beiträge zur Entomologie 49: 357-367.

Maus, C. 2000. Fourth taxonomical contribution to the subgenus Coprochara Mulsant \& Rey, 1874 of the genus Aleochara Gravenhorst, 1802. Description of four new species (Coleoptera: Staphylinidae). Koleopterologische Rundschau 70: 6978.

Maus, C. 2001. Redescription of Aleochara (Coprochara) signaticollis Fairmaire \& Germain, 1861, an overlooked species. $3^{\text {rd }}$ taxonomic contribution to the subgenus Coprochara Mulsant \& Rey, 1874 of the genus Aleochara Gravenhorst, 1802 (Coleoptera: Staphylinidae). Koleopterologische Rundschau 71: 37 48.

Maus, C.; B. Mittmann \& K. Peschke. 1998. Host Records of Parasitoid Aleochara Gravenhorst species (Coleoptera, Staphylinidae) attacking puparia of cyclorrhapheous Diptera. Deutsche Entomologische Zeitschrift 45: 231-254.

Mise, K.M.; L.M. Almeida \& M.O. Moura. 2007. Levantamento da fauna de Coleoptera que habita a carcaça de Sus scrofa L., em Curitiba, Paraná. Revista Brasileira de Entomologia 51: 358-368.

Motschulsky, V. 1858. Énumeration des nouvelles espèces de Coléoptères rapportés de ses voyages. Bulletin de la Société Impériale des Naturalistes de Moscou 31: 204-264.

Mulsant, E. \& C. Rey. 1874. Histoire naturelle de coléoptères de France. Brévipennes. Aléocharaires. Paris, 565p.

Muona, J. 1977. Nomenclatorial notes on Staphylinidae (Coleoptera). Notulae Entomologicae 57: 15-16.

Navarrete-Heredia, J.L.; A.F. Newton; M.K. Thayer; J.S. Ashe \& D.S. Chander. 2002. Guía ilustrada de los géneros de Staphylinidae (Coleoptera) de México - Illustrated Guide to the Genera of Staphylinidae (Coleoptera) of Mexico. México, Universidad de Guadalajara y CONABIO, XII+401p.

Newton, A.F. \& M.K. Thayer. 1992. Current classification and family-group names in Staphyliniformia (Coleoptera). Fieldiana: Zoology, New Series, 67: 1-92.

Newton, A.F. \& M.K. Thayer. 2005. Catalog of higher taxa of Staphyliniformia and genera and subgenera of Staphylinoidea. Chicago, Field Museum of Natural History, available online at http://www.fieldmuseum.org/peet_staph/ db_1a.html [accessed 14/III/2008].

Newton, A.F.; C.G. Chacón \& D.S. Chandler. 2005. Checklist of the Staphylinidae (Coleoptera) of Colombia. Biota Colombiana 6: 1-72.

PACE, R. 1987. Aleocharinae del Cile (Coleoptera Staphylinidae) (97 Contributo alla conoscenza delle Aleocharinae). Redia 70: 459-522.

PaCe, R. 1990. Nuovi Falagriini, Hoplandriini ed Aleocharini della Regione Neotropica (Coleoptera, Staphylinidae) (LXXX contributo alla conoscenza delle Aleocharinae). Giornale italiano d'Entomologia 5: 157-180.

PACE, R. 1999. Aleocharinae del Cile (Coleoptera, Staphylinidae) (114 ${ }^{\circ}$ Contributo alla conoscenza delle Aleocharinae). Bollettino del Museo Civico di Storia Naturale di Verona 23: $119-210$.

PACE, R. 2000. Aleocharinae del Cile, nuove o poco note (Coleoptera, Staphylinidae). $157^{\circ}$ Contributo alla conoscenza delle Aleocharinae. Bollettino del Museo Regionale di Scienze Naturali - Torino 17 (1999): 355-510.

PeschKe, K. \& D. Fuldner. 1977. Ubersicht und neue Untersuchungen zur Lebensweise der parasitoiden Aleocharinae (Coleoptera: Staphylinidae). Zoologische Jahrbucher: Abteilung fur Systematik, Okologie und Geographie der Tiere 104: 242-262.

SAHLBERG, R.F. 1844. Coleoptera diebus XV-XXVII Decembris anni DCCCXXXIX ad Rio Janeiro lecta. Acta Societatis Scientiarum Fennicae 2: 499-522.

SAY, T. 1832. Descriptions of new species of North American insects, and observations on some already described. New Harmony: T. Say, p. 50-57.

SAY, T. 1833. Descriptions of new species of North American insects, and observations on some already described. New Harmony, T. Say, p. 58-73.

SCheERPeltz, O. 1960. Zur Kenntnis neotropischer Staphyliniden (Col.). Beiträge zur Neotropischen Fauna 2: 65-138.

Scheerpeltz, O. 1972. Wissenschaftliche Ergebnisse der Studienreise von Gy. Topál nach Südwest-Argentinien (Coleoptera: Staphylinidae). Folia Entomologica Hungarica (S.N.) 25 (Suppl.): 1-268.

SHARP, D.S. 1874. The Staphylinidae of Japan. Transactions of the Entomological Society of London 1874: 1-103.

SHARP, D.S. 1876. Contribution to an insect fauna of the Amazon Valley. Coleoptera-Staphylinidae. Transactions of the Entomological Society of London 1876: 27-424.

ShARP, D.S. 1883. Staphylinidae. In: Biologia Centrali-Americana. Insecta. Coleoptera 1. London, Taylor \& Francis, p. 145-312.

Sharp, D.S. 1887. Staphylinidae. In: Biologia Centrali-Americana. Insecta. Coleoptera 1. London, Taylor \& Francis, p. 673-824.

Smetana, A. 2004. Subfamily Aleocharinae Fleming, 1821. In: L. LÖbl \& A. SMETANA (Eds). Catalogue of Palaearctic Coleoptera. Strenstrup, Apollo Books, vol. 2, p. 353-494.

Solier, A.J.J. 1849. Orden III. Coleopteros. In: C. GAY (Ed.). Historia fisica y politica de Chile. Zoologia. 4. Paris, C. Gay, p. 105-380.

Solsky, S.M. 1868. Études sur les Staphylinides de Méxique. Horae Societatis Entomologicae Rossicae 5: 119-144.

Solsky, S.M. 1870. Staphylins de l'Amérique méridionale et du Méxique. Bulletin de la Société Impériale des Naturalistes de Moscou 42: 257-267.

Solsky, S.M. 1872. Enumération et description des coléoptères de la famille des Staphylinides recueillis par Mrs. C. Jelsky 
et le Baron de Nolken pendant leurs voyages dans l'Amérique du Sud en 1870 et 1871 . Horae Societatis Entomologicae Rossicae 8: 289-314.

SouzA, A.M. DE \& A.X. Linhares. 1997. Diptera and Coleoptera of potential forensic importance in southeastern Brazil: relative abundance and seasonality. Medical and Veterinary Entomology 11: 8-12.

Sprague, P.S. 1870. A new rove-beetle: parasite on the cabbage maggot. American Entomologist 2: 370.

STEPHENS, J.F. 1832. Illustrations of British entomology; or, a synopsis of indigenous insects; containing their generic and specific distinctions; with an account of their metamorphoses, times of appearance, localities, food, and economy, as far as practicable. Mandibulata. 5.
London, Baldwin and Cradock, 240p.

TABOR, K.L.; R.D. Fell \& C.C. Brewster. 2005. Insect fauna visiting carrion in Southwest Virginia. Forensic Science International 150: 73-80.

Thomson, C.G. 1859. Skandinaviens Coleoptera, synoptiskt bearbetade. 1. Lund, Berlingska Boktryckeriet, 290p.

Thomson, C.G. 1860. Skandinaviens Coleoptera, synoptiskt bearbetade. 2. Lund, Berlingska Boktryckeriet, 304p.

Wollaston, T.V. 1854. Insecta Maderensia; being an account of the insects of the islands of the Madeiran group. London, J. Van Voorst, 634p.

Wright, E.; P. Mueller \& J. KeER. 1989. Agents for biological control of novel hosts: assessing an Aleocharine parasitoid of dungbreeding flies. Journal of Applied Ecology 26: 453-461.

Submitted: 05.V.2008; Accepted: 04.XII.2008.

Editorial responsibility: José Albertino Rafael 